\title{
Approximate Model of the Coaxial Pulsed Plasma Thruster
}

\author{
Victor V. Kuzenov ${ }^{1,2,3}$, Pavel A. Frolko ${ }^{1}$
}

${ }^{1}$ Department of Thermal Physics, Bauman Moscow State Technical University, Moscow, 105005, Russian Federation

${ }^{2}$ A.Yu. Ishlinsky Institute for Problems in Mechanics RAS, Moscow, 119526, Russian Federation

${ }^{3}$ N.L. Dukhov All-Russia Research Institute of Automatics, Moscow, 127055, Russian Federation

Keywords: High frequency ion source, RF low-pressure discharge, helicon discharge, mathematical model, ionization, non-uniform electric and magnetic fields.

Abstract. The aim of this work is to create an approximate mathematical model of a helicon type plasma source (coaxial pulsed plasma thruster) and calculation based on the developed multiparameter computational model.

\section{Introduction}

The aim of a given work is to build up close approximated mathematical model which will allow to compute main electrophysical characteristics of a coaxial magnetoplasma accelerator (CMPA) with the voluminous energy source and the system of preionisation of working gas on the basis of a helicon discharge [1-3].

\section{Mathematical model of excitation and absorption of eigenwaves in RF-ion source with a magnetic field}

Consider the first element of an approximated mathematical model of High Frequency (RF) Ion Source. This mathematical model describes processes connected with absorption and excitation of voluminous eigenwaves in RF ion source. We introduce the concept of electron $\omega_{H}$ and ion $\Omega_{H}$ gyrofrequency and plasma electron $\omega_{p}$ and ion $\Omega_{p}$ frequency accordingly. These values can be written as (1):

$$
\omega_{p}=\left(\frac{4 \pi n_{e} e^{2}}{m_{e}}\right)^{2}, \Omega_{p}=\left(\frac{4 \pi n_{i} e^{2}}{M_{i}}\right)^{2}, \omega_{H}=\frac{\left|e \overrightarrow{B_{0}}\right|}{m_{e} c}, \Omega_{H}=\frac{\left|e \overrightarrow{B_{0}}\right|}{M_{i} c},
$$

where $m_{e}$ and $M_{i}$ are electron and ion mass accordingly.

Helicon waves in a plasma of RF discharge (with the external magnetic field) can take place, when the following condition [4]: $\Omega_{H}, \omega_{L H}<\omega, \omega_{H}<\omega_{P}$ is fulfilled for the frequency $\omega$ of external power generator.

First, select the cylindrical coordinate system $(r, \varphi, z)$ with unite vectors $\left(\overrightarrow{r_{0}}, \overrightarrow{\varphi_{0}}, \overrightarrow{z_{0}}\right)$. These coordinates are connected with Cartesian coordinate relations:

$$
x=r \cos \varphi, r=\left(x^{2}+y^{2}\right)^{1 / 2}, \quad y=r \sin \varphi, \varphi=\arctan (y / x) .
$$

Maxwell's equations in cylindrical coordinates take the form:

$$
\begin{aligned}
& \frac{1}{r} \frac{\partial E_{z}}{\partial \varphi}-\frac{\partial E_{\varphi}}{\partial z}=--i k_{0} B_{r}, \frac{\partial E_{r}}{\partial z}-\frac{\partial E_{z}}{\partial r}=-i k_{0} B_{\varphi}, \frac{1}{r} \frac{\partial\left(r E_{\varphi}\right)}{\partial r}-\frac{1}{r} \frac{\partial E_{r}}{\partial \varphi}=-i k_{0} B_{z}, \\
& \frac{1}{r} \frac{\partial H_{z}}{\partial \varphi}-\frac{\partial H_{\varphi}}{\partial z}=i k_{0} D_{r}+\frac{4 \pi}{c} j_{r}, \frac{\partial H_{r}}{\partial z}-\frac{\partial H_{z}}{\partial r}=i k_{0} D_{\varphi}+\frac{4 \pi}{c} j_{\varphi}, \\
& \frac{1}{r} \frac{\partial\left(r H_{\varphi}\right)}{\partial r}-\frac{1}{r} \frac{\partial H_{r}}{\partial \varphi}=i k_{0} D_{z}+\frac{4 \pi}{c} j_{z},
\end{aligned}
$$

where $k_{0}=\omega / c$ is wave number in free space.

The equations of continuity may be written (4): 


$$
\frac{1}{r} \frac{\partial\left(r D_{r}\right)}{\partial r}+\frac{1}{r} \frac{\partial D_{\varphi}}{\partial \varphi}+\frac{\partial D_{z}}{\partial z}=4 \pi \rho, \frac{1}{r} \frac{\partial\left(r B_{r}\right)}{\partial r}+\frac{1}{r} \frac{\partial B_{\varphi}}{\partial \varphi}+\frac{\partial B_{z}}{\partial z}=0 .
$$

The relation between the transverse components of the vector in cylindrical and Cartesian coordinates is given by the ratio (5):

$$
\begin{aligned}
& A_{r}=A_{x} \cos \varphi+A_{y} \sin \varphi, A_{x}=A_{r} \cos \varphi-A_{\varphi} \sin \varphi, \\
& A_{\varphi}=-A_{x} \sin \varphi+A_{y} \cos \varphi, A_{y}=A_{r} \sin \varphi+A_{\varphi} \cos \varphi .
\end{aligned}
$$

Constitutive equations [5] are written in the following way (6):

$$
\vec{D}=\varepsilon \vec{E}, \vec{B}=\vec{H},
$$

where $\varepsilon$ is the tensor of dielectric permeability of magnetoactive plasma. Constitutive equations in such a form mean that value $H$ is identified with magnetic induction $B$.

Under mathematical description of physical processes in RF-ion source lets assume that solid medium present in gas discharge chamber is low temperature plasma, uniform external magnetic field $\frac{\overrightarrow{\partial B_{0}}}{\partial t}=0$ is directed towards axis $z$ of a cylindrical coordinate system. In this case the tensor of dielectric permeability of plasma $\varepsilon$ is (7):

$$
\varepsilon=\left(\begin{array}{ccc}
\varepsilon_{\perp} & i g & 0 \\
-i g & \varepsilon_{\perp} & 0 \\
0 & 0 & \varepsilon_{\|}
\end{array}\right) .
$$

Tensor components $\varepsilon$ B are set by (8) in a two-component plasma consisting [6] electrons and ions distributed according to Maxwell distribution, at the account of electron and ion neutral and electron-ion collisions in a plasma, ignoring electron-electron collisions (8):

$$
\begin{gathered}
\varepsilon_{\perp}=\varepsilon_{\perp}^{\mathrm{Re}}+i \varepsilon_{\perp}^{\mathrm{Im}}=1-\frac{\omega_{L e}^{2}\left(\omega+i v_{e}\right)}{\left[\left(\omega+i v_{e}\right)^{2}-\Omega_{e}^{2}\right] \omega}-\frac{\omega_{L i}^{2}\left(\omega+i v_{i}\right)}{\left[\left(\omega+i v_{i}\right)^{2}-\Omega_{i}^{2}\right] \omega}+ \\
+i \sqrt{\frac{\pi}{8}} \frac{\omega_{L e}^{2}}{\omega k_{z} V_{T e}} \exp \left(-\frac{\left(\omega-\Omega_{e}\right)^{2}}{2 k_{z}^{2} V_{T e}^{2}}\right)+i \sqrt{\frac{\pi}{8}} \frac{\omega_{L i}^{2}}{\omega k_{z} V_{T i}} \exp \left(-\frac{\left(\omega+\Omega_{i}\right)^{2}}{2 k_{z}^{2} V_{T i}^{2}}\right), \\
g=g^{\mathrm{Re}}+i g^{\mathrm{Im}}=-\frac{\omega_{L e}^{2} \Omega_{e}}{\left[\left(\omega+i v_{e}\right)^{2}-\Omega_{e}^{2}\right] \omega}+\frac{\omega_{L i}^{2} \Omega_{i}}{\left[\left(\omega+i v_{i}\right)^{2}-\Omega_{i}^{2}\right] \omega}+ \\
+i \sqrt{\frac{\pi}{8}} \frac{\omega_{L e}^{2}}{\omega k_{z} V_{T e}} \exp \left(-\frac{\left(\omega-\Omega_{e}\right)^{2}}{2 k_{z}^{2} V_{T e}^{2}}\right)-i \sqrt{\frac{\pi}{8}} \frac{\omega_{L i}^{2}}{\omega k_{z} V_{T i}} \exp \left(-\frac{\left(\omega+\Omega_{i}\right)^{2}}{2 k_{z}^{2} V_{T i}^{2}}\right), \\
+i \sqrt{\frac{\pi}{8}} \frac{\omega_{L e}^{2} \omega}{k_{z}^{3} V_{T e}^{3}} \exp \left(-\frac{\omega^{2}}{2 k_{z}^{2} V_{T e}^{2}}\right)+i \sqrt{\frac{\pi}{8}} \frac{\omega_{L i}^{2} \omega}{k_{z}^{3} V_{T i}^{3}} \exp \left(-\frac{\omega^{2}}{2 k_{z}^{2} V_{T i}^{2}}\right),
\end{gathered}
$$

where $\omega$ is the frequency of high frequency magnetic field emerging in a helicon plasma from antennas of different configurations, $\omega_{L i}, \omega_{L e}$ are the Langmuir frequencies of ions and electrons with densities $n_{i}$ and $n_{e}$, respectively, $\Omega_{e}, \Omega_{i}$ is the Larmor frequency of rotation of electrons and ions in the external magnetic field $B, V_{T e}, V_{T i}$ is the thermal velocity of the electrons and ions, $v_{e n}=n_{0} V_{T e} \sigma_{i o n}$ is the frequency of collisions (with ionization) of electrons with neutral particles, $n_{0}$ is the concentration of neutral particles in a plasma of helicon discharge, $\sigma_{i o n}$ is differential cross section of electron ionization of neutrals, $v_{e i, k}$ is the medium frequency of electro-ion collisions (with ion of the $k$ type) with the impulse transfer: $\tau_{e i}=\frac{1}{v_{e i}} \cdot \frac{(1+\theta)}{8}, E_{f}=\left(h^{2} / 2 m_{e}\right)\left(3 \pi^{2} n_{e}\right)^{2 / 3}$ is the 
energy of Fermi degeneration, $\theta=\exp \left(-E_{f} / k T_{e}\right)$ is the parameter of plasma degeneration, describing among other things limited cases - classical $(\theta \gg 1)$, and completely degenerate $(\theta=0)$ plasma, $n_{k}$ is the concentration of $k$ type ions.

The Coulomb logarithm considering the interaction of electrons and ions $\ln \Lambda_{e i}$ is defined by relations [7]. The medium frequency of ion-ion collisions is equal to:

$$
\begin{gathered}
v_{i i, k}=\frac{4 \sqrt{2 \pi} n_{k} Z_{i, k}^{2} e^{4} \ln \Lambda_{i}}{3 M_{i, k}^{1 / 2}\left(k T_{i, k}\right)^{3 / 2}}, \\
\tau_{i i}=\frac{1}{v_{i i}}, \ln \Lambda_{i}=0,5 \ln \left[1+\frac{4,3993 \cdot 10^{4} \cdot T_{i}^{3}}{\rho}\right], T[\mathrm{keV}], \rho\left[\mathrm{g} / \mathrm{cm}^{3}\right] .
\end{gathered}
$$

Collision and non-collision (Cherenkov and cyclotron) absorption of electromagnetic waves in a plasma are taken into account in tensor (8) of a dielectric permeability of a plasma $\varepsilon$. The exponential items describe the absorption of voluminous eigenwaves in a plasma using noncollision Landau mechanism, and the other items correspond to collision absorption of electromagnetic waves. Maxwell's equations in cylindrical coordinates (3), (4) with the account of a special type (7), (8) of dielectric plasma permeability $\varepsilon$ and $\frac{\partial}{\partial \varphi} \equiv 0$ take the form (10):

$$
\begin{array}{r}
-\frac{\partial E_{\varphi}}{\partial z}=-i k_{0} B_{r}, \quad \frac{\partial E_{r}}{\partial z}-\frac{\partial E_{z}}{\partial r}=-i k_{0} B_{\varphi}, \quad \frac{1}{r} \frac{\partial\left(r E_{\varphi}\right)}{\partial r}=-i k_{0} B_{z}, \\
-\frac{\partial H_{\varphi}}{\partial z}=i k_{0} D_{r}+\frac{4 \pi}{c} j_{r}, \frac{\partial H_{r}}{\partial z}-\frac{\partial H_{z}}{\partial r}=i k_{0} D_{\varphi}+\frac{4 \pi}{c} j_{\varphi}, \quad \frac{1}{r} \frac{\partial\left(r H_{\varphi}\right)}{\partial x}=i k_{0} D_{z}+\frac{4 \pi}{c} j_{z} .
\end{array}
$$

Assume for analytical solution of equations system (10) that all the coefficients of this system are close to the coefficients calculated by thermodynamic parameters of plasma received by averaging the volume of gas discharge chamber of a helicon discharge. We simplify the solution of Maxwell's equations, taking only one particular member of Fourier series (one mode approximation) on $z$ coordinate: $\vec{E}(r, \varphi, z, t)=\vec{E}(r) \exp \left[-i \omega t+i k_{z} z\right], \vec{B}(r, \varphi, z, t)=\vec{B}(r) \exp \left[-i \omega t+i k_{z} z\right], k_{z}=\frac{\pi n}{L}$.

Following the work [8] it is possible to find the solution of the equation (11). To calculate the power emitted in a plasma of a helicon discharge $W_{\text {hel }}$ we use the following formula (11):

$$
W_{H e l}=\frac{L \omega}{4} \int_{0}^{R} r\left\{\varepsilon_{\perp}^{\mathrm{Im}}\left(\left|E_{r}\right|^{2}+\left|E_{\varphi}\right|^{2}\right)+\varepsilon_{\|}^{\mathrm{Im}}\left|E_{z}\right|^{2}+i g^{\mathrm{Im}}\left(E_{\varphi} E_{r}^{*}-E_{r} E_{\varphi}^{*}\right)\right\} d r .
$$

Active resistance of an antenna is defined on the basis of correlation: $R_{A}=\frac{L_{A n t}}{2 \pi R \delta \sigma_{0}}$, where $L_{A n t}$ is the antenna length along its constant, $\sigma_{0}$ is the electroconductivity of antenna material, $\delta$ is the classical width of a skin-layer, $R$ is the radius of a discharge chamber of helicon discharge.

Total antenna resistance should consider the phenomenon of self induction:

$R_{A n t}=\sqrt{R_{A}^{2}+\omega^{2} L_{e f}^{2}}$, where $L_{e f}$ is the equivalent antenna induction.

The second element of approximated mathematical model of RF ion source is fully described in work [9]. This element allows to find mathematical connections $\left(P_{h e l}=I_{e c}^{2} R_{A n t}+\alpha W_{h e l}\right)$ between the power $W_{\text {hel }}$ inserted into the plasma of a helicon discharge ( $P_{h e l}$ is the power supplied to the antenna) and termophysical parameters of a plasma.

\section{Mathematical model}

Accelerating channel of a coaxial magnetoplasma accelerator (CMPA) is built on the principle "pipe in the pipe", i.e. represents spatial area between two pipes of different diameter (cylindrical and central electrodes) which are situated one inside the other. 
Cylindrical electrode has the shape of a pipe of a constant cross section and possesses a bigger diameter $\left(2 R_{2}\right)$ than a central electrode (placed at the axis of a symmetry) which is also a pipe, but of a smaller diameter $\left(2 R_{1}\right)$. Besides, the construction of the accelerator includes solenoid (inductor) consisting of a system of turns (with the diameter $2 R_{l}$ ) with a circular transverse cross section.

The plasmoid is created by feeding voltage $U_{0}$ and following cut between cylindrical and central gas electrodes coming into accelerating CMPA. Then, plasmoid by electromagnetic method is compressed and accelerated (in the direction of symmetry axis). Under this, electrodynamic acceleration formed in an accelerated channel of a plasmoid, based on the interaction (described by Ampere's law) of magnetic field of an electric circuit with current carrying plasmoid.

The calculation of an electromagnetic force $F$, acting on the accelerated plasma is performed on the basis of a general expression given in the paper [10] with the usage of current forces $J_{j}, J_{i}$, flowing arbitrary circuits in the system "CMPA - accelerated plasma" (12):

$$
F_{q}=\frac{1}{2} \sum_{i, j} J_{i} J_{j} \frac{\partial L_{i j}}{\partial q}
$$

where $J_{j}, J_{i}$ are the currents flowing in $j$ and $i$ circuits, $L_{i j}$ is the coefficient of mutual induction $j$ and $i$ is the circuits, $q$ is the generalized coordinate.

Within the framework of made assumptions expression (12) can be written as:

$$
F_{q}=\frac{1}{2} J^{2} \sum_{i j} \frac{\partial L_{i j}}{\partial z}=\frac{1}{2} J^{2} \frac{\partial L}{\partial z},
$$

where $J$ is the current in a circular turn of the inductor or, which is the same, current in an electric circuit of the acceleration system, $L$ is the sum inductivity of the whole electromagnetic acceleration system of CMPA.

The original equation system, corresponding to a model configuration describing the dynamics of electromagnetic acceleration (with ideally conducting features) with the usage of a capacitor energy accumulator $C_{0}$ :

- the equation of electrotechnical circuit (14):

$$
U=R J+\frac{d}{d t}(L J), \frac{d U}{d t}=-\frac{J}{C_{0}} .
$$

- the equation of the accelerated plasmoid (15):

$$
M \frac{d V}{d t}=\frac{J^{2}}{2}\left(\frac{d L}{d z}\right)-P_{2} \pi\left(R_{2}^{2}-R_{1}^{2}\right), \frac{d z}{d t}=V .
$$

The following designations are introduced here: $C_{0}$ is the capacity of condenser power source of the system; $U$ is the voltage at capacitor accumulator at the time $t ; L=L_{0}+L_{1}+L_{2}+L_{3}$ is the sum inductivity of the equivalent electrotechnical circuit including constants $L_{0}$ (inductivity of capacitor accumulator and feeding bus bars), $L_{l}$ (sum turn inductivity with the current coming into the inductor), $L_{2}$ (summed mutual inductivity of all $i$ and $j$ circuits comprising the inductor), $L_{3}(t)$ is the inductivity of CMPA system variable in time; $R=R_{0}+R_{\text {ind }}$ is the summed active resistance of feeding bus bars $\left(R_{0}\right)$ and inductor $\left(R_{\text {ind }}\right) ; d z / d t=V, M(t)$ is the velocity and mass of the accelerated plasmoid.

Use the formula (16) to calculate the inductivity $L_{4}(t)$ of coaxial system and its linear inductivity $l$ (inductivity of a length unit of CMPA) [11]:

$$
L_{3}(t)=\ell z, \ell=\frac{\mu_{0} J^{2}}{4 \pi} \ln \left(\frac{R_{2}}{R_{1}}\right),
$$

where $R_{2}, R_{1}$ are the radii of central and external electrodes correspondingly, $\mu_{0}=4 \pi \cdot 10^{-7}[\mathrm{Hn} / \mathrm{m}]$ is the magnetic permeability of the vacuum, $z$ is the longitudinal coordinate CMPA along which an accelerated is disseminated.

\section{Some results of physical characteristics of RF source}


The experimental [12] and computing dependences of effective resistance of plasma $R_{\text {eff }}$ on the value of external magnetic field $B_{0}$, received using combined version of computer code are given in Fig. 1. The operating frequency of the antenna 13,56 MHz, gas Ar: $P=5$ mTorr, $R=7,5 \mathrm{~cm}, L=20$ $\mathrm{cm}$. To receive practically necessary spatial distribution of ion current density $j$, temperatures $T_{e}, T_{i}$, electron and ion densities in gas discharge chamber and the thrust, it is necessary to develop more exact models of RF plasma source.

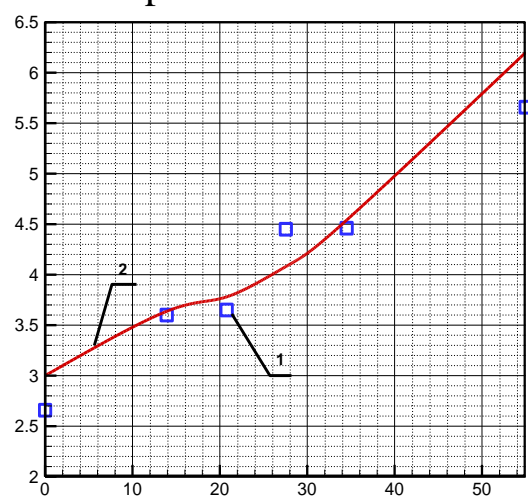

$R_{\text {eff, }},[\mathrm{ohm}]$

a) $P_{\text {hel }}=100 \mathrm{~W}$

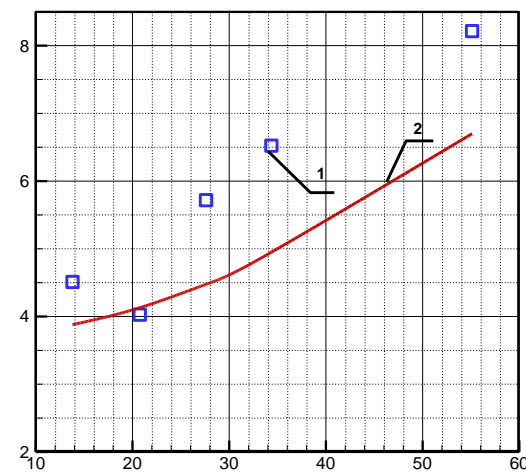

b) $P_{\text {hel }}=150 \mathrm{~W}$
$B_{0},[\mathrm{Gs}]$

Fig.1: Comparison of experimental (1) and calculated (2) dependences of the effective plasma resistance $R_{\text {eff }}$ on the external magnetic field $B_{0}$

\section{Conclusions}

The approximated mathematical model of high radio frequency ion source of a helicon type plasma and coaxial multi plasma accelerator is presented. Such devices can be used for high temperature plasma applications [13-18]. The initial verification of computing results on the basis of a group of experimental data is done. These computations were done with the use of self-approved approximated mathematical model of RF plasma source. The estimate of various power types contributions into the process of acceleration and transformation of one type of energy into the other is performed. Analyzed RF installations can be used as the thrusters for orbital correction and for geostationary or low orbit spacecrafts.

\section{Acknowledgements}

The research was partially conducted in the frame of fundamental research program of the RAS and supported by the Russian Ministry of Education and Science (Project No. 13.79.2014/K).

\section{References}

[1] Kerdtongmee P., Srinoum D. \& Nisoa M. Development of a compact permanent magnet helicon plasma source for ion beam bioengineering. Review of Scientific Instruments, 82, 103503, 2011.

[2] Sijoy C.D., Chaturvedi S. An Eulerian MHD model for the analysis of magnetic flux compression by expanding diamagnetic fusion plasma sphere. Fusion Engineering and Design, 87, pp. 104-117, 2012.

[3] Tarey R.D., Sahu B.B. \& A. Ganguli. Understanding helicon plasmas. Physics of Plasmas, 19, $073520,2012$.

[4] Bowers R., Legendy C. \& Rose F. Oscillatory galvanomagnetic effect in metallic sodium. Phys. Rev. Lett., 7, pp. 339-341, 1961.

[5] Harding G.N., Thonemann P.C. A study of helicon waves in indium. Proc. Phys. Soc., 85, pp. 317-328, 1965.

[6] Boswell R.W. Very efficient plasma generation by whistler waves near the lower hybrid frequency. Plasma Phys. Contr. Fusion, 26, pp. 1147, 1984.

[7] Chen F.F. Plasma ionization by helicon waves. Plasma Physics and Controlled Fusion, 33, pp. 339-364, 1991. 
[8] Boswell R.W., Chen F.F. Helicons, the early years. IEEE Trans. Plasma Sci., 25, pp. 1229-1244, 1997.

[9] Kuzenov V.V. Fiziko-khimicheskaya kinetika v gazovoi dinamike, 15, 2014. http://chemphys.edu.ru/media/files/11-30-002-.pdf (in Russian).

[10] Landau L.D., Lifshitz E.M. Electrodynamics of continuous media. Oxford: Pergamon Press, 1984.

[11] Kalantarov P.L., Tseitlin L.A. Raschet inductivnostei. Moscow: Energoatomizdat, 1986 (in Russian).

[12] Kral'kina E.A. Low-pressure RF inductive discharge and possibilities of optimizing inductive plasma sources. Phys. Usp., 51, pp. 493-512, 2008.

[13] Ryzhkov S.V. Modeling and engineering applications for weakly turbulent plasma. Proc. 35th EPS Conf. on Plasma Physics and Contr. Fusion, 32D, P1.114, 2008.

[14] Chirkov A.Yu., Ryzhkov S.V. The plasma jet/laser driven compression of compact plasmoids to fusion conditions. Journal of Fusion Energy, 31, pp. 7-12, 2012.

[15] Kuzenov V.V., Ryzhkov S.V. Radiation-hydrodynamic modeling of the contact boundary of the plasma target placed in an external magnetic field. Applied Physics, № 3, pp. 26-30, 2014.

[16] Kuzenov V.V., Ryzhkov S.V. Numerical modeling of magnetized plasma compressed by the laser beams and plasma jets. Problems of Atomic Science and Technology, № 1 (83), pp. 12-14, 2013.

[17] Kuzenov V.V., Ryzhkov S.V. Evaluation of hydrodynamic instabilities in inertial confinement fusion target in a magnetic field. Problems of Atomic Science and Technology, № 4 (86), pp. 103-107, 2013.

[18] Kuzenov V.V., Ryzhkov S.V., Shumaev V.V. Application of Thomas-Fermi model to evaluation of thermodynamic properties of magnetized plasma. Problems of Atomic Science and Technology, №. 1(95), pp. 97-99, 2015. 